\title{
Accuracy of rigid registration between amyloid / tau PET and MRI based on normalized mutual information
}

Yuma Iwao ( $\sim$ iwao.yuma@qst.go.jp )

National Institute of Radiological Sciences

Go Akamatsu

National Institute of Radiological Sciences

Kei Wagatsuma

Tokyo Metropolitan Institute of Gerontology

Muneyuki Sakata

Tokyo Metropolitan Institute of Gerontology

Kenji Ishii

Tokyo Metropolitan Institute of Gerontology

Miwako Takahashi

National Institute of Radiological Sciences

Taiga Yamaya

National Institute of Radiological Sciences

Original article

Keywords: amyloid PET, tau PET, MRI, registration, NMI, SPM

Posted Date: February 14th, 2020

DOI: https://doi.org/10.21203/rs.2.23496/v1

License: (9) This work is licensed under a Creative Commons Attribution 4.0 International License.

Read Full License 


\title{
Accuracy of rigid registration between amyloid / tau PET and MRI based on normalized mutual information
}

\author{
Yuma Iwao¹, Go Akamatsuㄹ, Kei Wagatsuma², Muneyuki Sakata², Kenji \\ Ishii $^{2}$, Miwako Takahashi ${ }^{1}$, Taiga Yamaya ${ }^{1}$
}

1 National Institute of Radiological Sciences, National Institutes for Quantum and Radiological Science and Technology

${ }^{2}$ Research Team for Neuroimaging, Tokyo Metropolitan Institute of Gerontology

\section{Declarations}

\section{Ethics approval and consent to participate}

All procedures performed in studies involving human participants were in accordance with the ethical standards of the institutional and/or national research committee and with the 1964 Helsinki declaration and its later amendments or comparable ethical standards. This study was approved by the ethics committee of the Tokyo Metropolitan Institute of Gerontology.

\section{Consent for publication}

Informed consent was obtained from all individual participants included in the study.

\section{Availability of data and material}

The datasets generated for this work and which support the findings are available on reasonable request.

\section{Competing interests}

The authors declare that they have no potential conflicts of interest.

\section{Funding}

This study was supported by ATOX Co., Ltd., Japan.

\section{Authors' contributions}


YI wrote the manuscript. YI, GA and MT analyzed the data. KW, MS, KI, and TY made substantial contributions to the conception and design of the study. 


\section{Abstract \\ Purpose}

Fusion images of positron emission tomography (PET) and magnetic resonance image (MRI) are effective to evaluate pathological changes using anatomical information. Registration of PET and MRI based on normalized mutual information (NMI) is widely used, where PET images change a lot depending on not only the choice of tracers but also disease progression, but the co-registration accuracy has not been confirmed well yet. The aim of this study is to clarify the accuracy of NMI-based registration between MRI and PET (amyloid PET and tau PET).

\section{Methods}

We obtained PET and MRI data from 69 participants (ages from 47 to 87) who underwent PET using one of the following four tracers: ${ }^{11} \mathrm{C}-\mathrm{PiB},{ }^{18} \mathrm{~F}$-Florbetapir, ${ }^{11} \mathrm{C}-\mathrm{PBB} 3$ or ${ }^{18} \mathrm{~F}-$ THK5351. The ground truth (i.e., a set of registered MRI-PET images) was manually made by two experts. For each data set, 40 set of perturbed parameters were given to MR images. Then each perturbed MRI was registered to the PET image using the statistical parametric mapping (SPM) tool. The differences between the given and the estimated shifts and rotates were calculated as registration errors.

\section{Results}

We found the registration errors were around $2 \mathrm{~mm}$ in the shift and around $1 \mathrm{deg}$ in the rotation. These registration errors were independent of the amount of the perturbation given.

\section{Conclusion}

Although negative subjects of tau tracers showed higher error than other tracers, the registration error was less than PET spatial resolution, which supported a proof of the stability of the SPM.

243 words / 250 words

Keyword amyloid PET, tau PET, MRI, registration, NMI, SPM 


\section{Introduction}

Detailed investigations of pathophysiology in persons who are suspected of having neuronal degenerative diseases or in persons who are experiencing cognitive impairment is indispensable for such activities as constructing care facilities, planning national healthcare budgets and planning medical treatment strategies. Recently, positron emission tomography (PET) with amyloid and tau tracers is being utilized to reveal pathological changes in the brain before the onset of significant clinical symptoms [1][2]. On the other hand, magnetic resonance imaging (MRI) is an essential tool to visualize brain anatomical structures. Therefore, a fusion image of PET and MRI is required to provide useful diagnostic information. To make the fusion image, an appropriate image registration of PET and MRI is needed that considers differences in voxel size, patient position and posture. As a general method of image registration, a registration function based on normalized mutual information (NMI) [3, 4] implemented in image analysis software such as the statistical parametric mapping (SPM) [5] is often used. The SPM has been widely applied for amyloid and tau PET image analysis and for voxel-based statistical comparisons [6-11]. It is important to understand the registration accuracy in order to use the software appropriately because PET images change a lot depending on not only the choice of tracers but also disease progression in dementia PET. One study about registration accuracy of amyloid PET and MRI using SPM has been reported by Schwarz CG, Jones DT, Gunter JL, Lowe VJ, Vemuri P, Senjem ML, et al. [12]. They analyzed the registration accuracy for some groups classified by clinical disease severity.

In this study, using the SPM function, we evaluated the accuracy of NMI based registration of amyloid PET and tau PET onto MRI images. In addition, we investigated the change in registration accuracy depending on amyloid and tau visual classifications (positive and negative).

\section{Data and Method}

\subsection{Registration method based on NMI}

NMI is widely used for evaluation values of similarity between two images. In the SPM registration, we can choose evaluation functions such as mutual information, NMI, the entropy correlation coefficient [13] and the normalized cross correlation. Among them, we chose NMI as it is a standard setting in SPM and it is the most widely used evaluation value in multi modal medical image registration.

Mutual information is a value that calculates entropy from the appearance frequency of pixel values in an image and evaluates the common entropy between two images that 
show similarity. NMI is the normalized mutual information; when there is no similarity in two images, the NMI value becomes 0 . When two images are completely the same, the NMI value becomes 1 .

\subsection{Imaging protocol}

In this study, we used PET images with four tracers. Two were amyloid PET tracers ${ }^{11} \mathrm{C}-\mathrm{PiB}[15]$ and ${ }^{18} \mathrm{~F}$-florbetapir $\left.[16,17]\right)$ and the other two were tau PET tracers ${ }^{11} \mathrm{C}$ PBB3 [18] and 18F-THK5351 [19]). Characteristics of individuals obtained by tracers are shown in Table 1. Each dataset was the paired PET and MRI data of the same individual. PET images were visually classified into amyloid positive or negative based on amyloid PET, and tau positive or negative based on tau PET as done by a physician specializing in nuclear neuroimaging. In the tau classification of tau PET images, a PET image with at least one abnormal uptake in any brain region was defined as positive. All PET data were acquired using the Discovery PET-CT 710 (GE Healthcare, Milwaukee, WI). PET images were reconstructed using an iterative reconstruction algorithm (3D-ordered subset expectation maximization) with time of flight (TOF) information (4 iterations, 16 subsets). Voxel size of PET images was $2 \mathrm{~mm} \times 2 \mathrm{~mm} \times$ $3.27 \mathrm{~mm}$, matrix size was $128 \times 128$ (256 mm field of view). Attenuation, scatter and random corrections and a $4 \mathrm{~mm}$ Gaussian post filter were applied. Total dose, uptake time, scan time of each subject are shown in supplemental Table S1. All MRI are T1weighted images, details of imaging conditions are shown in supplemental Table S2.

\subsection{Evaluation process of the registration accuracy}

The flow of registration accuracy evaluation is shown in Fig. 1. The detailed steps (a-d) in the figure are described below. The SPM version we used in this study was SPM12.

\section{Step (a): Preprocessing}

In order to create the reference image, first, the voxel size and field of view (FOV) were converted to those of the PET image. Second, the MRI was moved and rotated manually using in-house software for strict matched by one nuclear medicine physician and one nuclear medicine technologist. The software displayed the PET image and MR image individually or with overlapping by using different color scales and different view directions, such as the axial, coronal, and sagittal image views. This registered MR image and its corresponding PET image were used as the reference images for the registration accuracy evaluation. Representative fusion images of registered MRI and PET are shown in Fig. 2. 
Step (b): Creating perturbed MRI

Reference data of the MRI which was made in step (a) was transformed by 40 sets of 6 different perturbed parameters. The base and maximum values of the perturbed parameters are shown in Table 2. These values were derived from the head motion evaluation in a FDG-PET dynamic study [20]. Each perturbed parameter was randomly varied in the range from the base value to the maximum value.

Step (c): Automatic registration according to NMI

MRI transformed by the parameter in step (b) and the PET image were registered by SPM function. In this step, we used the default setting of the SPM software, the cost function was the NMI, the optimization sampling interval was $4 \mathrm{~mm}$ first and after that $2 \mathrm{~mm}$, tolerances for accuracy of each parameter were, $x=0.02, y=0.02, z=0.02$, $\operatorname{rot} x=0.001, \operatorname{rot} y=0.001, \operatorname{rot} z=0.001$, and applied we smoothing to the $256 \times 256$ joint histogram which is needed for NMI as $7 \times 7$ voxels.

Step (d): Error evaluation

The amounts of translation and rotation were defined as follows:

Translation parameters were defined for each axis, $\mathrm{x}$ (right-left), y (anterior-posterior), $\mathrm{z}$ (superior-inferior) directions, and rotation parameters were defined around each axis $(\operatorname{rot} \mathrm{x}, \operatorname{rot} \mathrm{y}, \operatorname{rot} \mathrm{z})$. The amount of translation $s$ was calculated by the following equation. $s=\sqrt{x^{2}+y^{2}+z^{2}}$.

The three angles ( rot x, rot y, rot z) are called Euler angles and they represent orientation of the rigid model. These 3 parameters can be to converted into a three-dimensional rotation axis vector and the amount of rotation around it. These 4 parameters (rot $\mathrm{x}$, rot $\mathrm{y}, \operatorname{rot} \mathrm{z}$ and the amount of rotation) are called a quaternion [14], and the amount of rotation $r$ was calculated as follows:

$$
r=\arccos (\cos (\phi) \times \cos (\theta) \times \cos (\psi)+\sin (\phi) \times \sin (\theta) \times \sin (\psi)) \times 2,
$$

where $\phi=\operatorname{rot} x / 2, \theta=\operatorname{rot} y / 2, \psi=\operatorname{rot} z / 2$.

We evaluated the differences of the registration result parameters from step (c) and the selected perturbed parameters in step (b), and used it as the error of the automatic registration. The amount of error was calculated as the pair of $s\left(\bar{x}-x_{0}, \bar{y}-y_{0}, \bar{z}-z_{0}\right)$ and $r\left(\bar{\phi}-\phi_{0}, \bar{\theta}-\theta_{0}, \bar{\psi}-\psi_{0}\right)$, where $(\bar{x}, \bar{y}, \bar{z})$ and $(\bar{\phi}, \bar{\theta}, \bar{\psi})$ are the result parameters of transformation and rotation, respectively and $\left(x_{0}, y_{0}, z_{0}\right)$ and $\left(\phi_{0}, \theta_{0}, \psi_{0}\right)$ are the perturbed parameters of transformation and rotation, respectively. 


\subsection{Data Analysis}

We examined 40 patterns of 6 perturbed parameters in each of 69 subjects; hence, we examined a total of 2760 patterns. Welch's $t$ test [21] was performed to find any statistically significant difference between the classification of the amyloid or tau PET images. A $p$ value of $t$-test less than 0.05 was considered statistically significant.

\section{Results}

The registration errors for each tracer are shown in Fig. 3. The results were represented as box plots to visualize the amount of the registration error. All box plots had whiskers which extended from the hinge to the highest and lowest values within $1.5 \times$ the interquartile range (IQR). Notches represented a $95 \%$ confidence interval around the median [22]. The median values of translation error were less than $1 \mathrm{~mm}$ except for $\mathrm{y}$-axis and z-axis of PBB3 negative subjects. The median values of rotation error were about 1 deg.

The perturbed parameter amounts are plotted against the registration error in Fig. 4 as a true value. There was no correlation for any of the tracers. In the translation, standard deviations of registration error were $0.01-0.28 \mathrm{~mm}$, and in the rotation, standard deviations were 0.01-0.42 deg.

\section{Discussion}

We evaluated the accuracy of the registration method based on NMI between amyloid or tau PET and MRI. The accuracy of the registration between amyloid PET and MRI has been reported by other groups; however, to our knowledge, this is the first study to evaluate the accuracy of the registration between tau PET and MRI.

As shown in Fig. 3, the median of translation error was smaller than $2 \mathrm{~mm}$, and the median of rotation error was around $1 \mathrm{deg}$ for all tracers, which corresponded to about 2 $\mathrm{mm}$ error in maximum at the edge of the FOV. Among all tracers, the error in the axial direction was relatively larger than that in other directions. This was possibly because the PET voxel size in the $\mathrm{z}^{-}$axis direction $(3.27 \mathrm{~mm})$ was larger than that in the $\mathrm{x}^{-}$and $\mathrm{y}$-axis directions $(2 \mathrm{~mm})$. This result indicates that the registration is possible with an error smaller than 1 PET voxel size, which was $2 \mathrm{~mm} \times 2 \mathrm{~mm} \times 3.27 \mathrm{~mm}$. 
In the PBB3 negative images, we found larger translation errors in the $\mathrm{z}$-axis direction than other directions. This might be due to the retention of high radioactivity in the venous sinus because of rapid metabolism of PBB3 (Fig. 2) [18, 23]. These extra-brain uptakes were more noticeable than brain uptakes, which potentially could affect the registration accuracy, especially in the z-axis direction. It is reasonable that the signals corresponding to these non-specific accumulations in PET do not appear in MRI; therefore, evaluation values got worse and that made the registration incorrect. On the other hand, as mentioned above, all errors were less than $2 \mathrm{~mm}$ or around $1 \mathrm{deg}$, and it is suggested that the registration error is less than 1 PET voxel order. We conclude that the registration accuracy of NMI is enough for making PET and MRI fusion images.

In addition, registration errors were compared between positive and negative images. There were significant differences among all tracers. In the amyloid tracers ( $\mathrm{PiB}$ and florbetapir), the registrations for negative images were slightly more accurate than those for positive images. For amyloid negative images, the contrast between white and gray matter was higher than amyloid positive images. White and gray matter are areas with high contrast in MRI. Therefore, if higher contrast between white and gray matter in the PET image is shown, a better value of NMI will be obtained. Therefore, it is reasonable that MRI was more correctly registered to amyloid negative images than to amyloid positive images. Schwarz et al. [12] indicated that Alzheimer's disease had a larger registration error than the control group in amyloid PET images, and our results are consistent with their report.

For the tau tracers (PBB3 and THK5351), positive images were slightly more accurate than negative images. In the negative images, the contrast between white and gray matter was low (Fig. 2). This indicates that the NMI value will be poor between PET and MRI. This was the reason for the low accuracy in negative images. Furthermore, it is known that the THK5351 tracer exhibits off-target binding of monoamine oxidase B [24]. Uptakes in the basal ganglia would be high especially in the negative case (Fig. 3). However, the contrast of the basal ganglia was relatively low in the T1-weighted MRI. These contrast mismatches between THK5351-PET and MRI might affect the accuracy of registration.

Fig. 4 plots the perturbed parameter amount vs. the error amount. The same color dots (which indicate data for the same subject) showed nearly constant errors regardless of the perturbed amounts. As previously mentioned, standard deviations of registration 
error were $0.01-0.28 \mathrm{~mm}$, and in the rotation, standard deviations were 0.01-0.42 deg, these indicate that $95 \%$ of the registration errors were included at about $\pm 0.5 \mathrm{~mm}$ or $\pm 0.8 \mathrm{deg}$ from each average value. This was much smaller than 1 PET voxel size, therefore in other words, registration error converged to fixed results regardless of the amount of the perturbation in the initial position of registration in PET and MRI. In the SPM, cost function (NMI) optimization is done twice at different resolutions. In the standard setting, the first images are resized to a $4 \mathrm{~mm}$ size voxel, and the registration process is done for these images. The second images are resized to a $2 \mathrm{~mm}$ size voxel and registration is done again. Initial parameters of the second registration use the first registration results. These two registration processes mean a wide search range is possible, and this leads to high convergence.

This study included subjects clinically diagnosed with Alzheimer's disease, progressive supranuclear palsy, corticobasal degeneration, frontotemporal lobar degeneration, and argyrophilic grain dementia. The subjects showed various brain uptake patterns in amyloid or tau PET and had various levels of brain atrophy. From the viewpoint of clinical application, the present subject group would be suitable for evaluating the accuracy of PET and MRI registration.

\section{Conclusion}

In this work, we evaluated the accuracy of the NMI-based MRI registration to amyloid or tau PET images. For the tau tracers (PBB3 and THK5351), tau-negative images especially had a higher registration error than other conditions. However, registration results based on NMI using SPM were converged around 1 PET voxel from manual registration. Furthermore, our registration results had high reproducibility, and the NMI based registration did not depend on the initial registration position. 


\section{References}

1. Rowe CC, Villemagne VL. Brain Amyloid Imaging. J Nucl Med Technol. 2013;41:11-8.

2. Villemagne VL, Fodero-Tavoletti MT, Masters CL, Rowe CC. Tau imaging: early progress and future directions. Lancet Neurol. 2015;14:114-24.

3. Viola P, Wells III WM. Alignment by Maximization of Mutual Information. Int J Comput Vis. 1997;24:137-54.

4. Hill DL, Batchelor PG, Holden M, Hawkes DJ. Medical image registration. Phys Med Biol. 2001;46:R1-45.

5. Friston KJ, Holmes AP, Worsley KJ, Poline J-P, Frith CD, Frackowiak RSJ. Statistical parametric maps in functional imaging: A general linear approach. Hum Brain Mapp. 1994;2:189-210.

6. Kemppainen NM, Aalto S, Wilson IA, Nagren K, Helin S, Bruck A, et al. Voxel-based analysis of PET amyloid ligand ${ }^{11} \mathrm{C}$ PIB uptake in Alzheimer disease. Neurology. 2006;67:1575-80.

7. Shin J, Lee S-Y, Kim SJ, Kim S-H, Cho S-J, Kim Y-B. Voxel-based analysis of Alzheimer's disease PET imaging using a triplet of radiotracers: PIB, FDDNP, and FDG. Neuroimage. 2010;52:488-96.

8. Sojkova J, Goh J, Bilgel M, Landman B, Yang X, Zhou Y, et al. Voxelwise Relationships Between Distribution Volume Ratio and Cerebral Blood Flow: Implications for Analysis of -Amyloid Images. J Nucl Med. 2015;56:1042-7.

9. Schöll M, Lockhart SN, Schonhaut DR, O’Neil JP, Janabi M, Ossenkoppele R, et al. PET Imaging of Tau Deposition in the Aging Human Brain. Neuron. 2016;89:971-82.

10. Baker SL, Lockhart SN, Price JC, He M, Huesman RH, Schonhaut D, et al. Reference Tissue-Based Kinetic Evaluation of 18 F-AV-1451 for Tau Imaging. J Nucl Med. 2017;58:332-8.

11. Jang YK, Lyoo CH, Park S, Oh SJ, Cho H, Oh M, et al. Head to head comparison of [18F] AV-1451 and [18F] THK5351 for tau imaging in Alzheimer's disease and frontotemporal dementia. Eur J Nucl Med Mol Imaging. 2018;45:432-42.

12. Schwarz CG, Jones DT, Gunter JL, Lowe VJ, Vemuri P, Senjem ML, et al. Contributions of imprecision in PET-MRI rigid registration to imprecision in amyloid PET SUVR measurements. Hum Brain Mapp. . Hum Brain Mapp. 2017; Jul;38(7) 3323-36. doi: $10.1002 / \mathrm{hbm} .23622$

13. Maes F, Collignon A, Vandermeulen D, Marchal G, Suetens P. Multimodality image registration by maximization of mutual information. IEEE Trans Med Imaging. 1997;16(2):187-98. doi: 10.1109/42.563664

14. Grubin C. Derivation of the quaternion scheme via the Euler axis and angle. J Spacecr 
Rockets. 1970;7:1261-3.

15. Klunk WE, Engler H, Nordberg A, Wang Y, Blomqvist G, Holt DP, et al. Imaging brain amyloid in Alzheimer's disease with Pittsburgh Compound-B. Ann Neurol. 2004;55:306-19. 16. Wong DF, Rosenberg PB, Zhou Y, Kumar A, Raymont V, Ravert HT, et al. In Vivo Imaging of Amyloid Deposition in Alzheimer Disease Using the Radioligand 18F-AV-45 (Flobetapir F 18). J Nucl Med. 2010;51:913-20.

17. Clark CM, Pontecorvo MJ, Beach TG, Bedell BJ, Coleman RE, Doraiswamy PM, et al. Cerebral PET with florbetapir compared with neuropathology at autopsy for detection of neuritic amyloid-B plaques: a prospective cohort study. Lancet Neurol. 2012;11:669-78. 18. Maruyama M, Shimada H, Suhara T, Shinotoh H, Ji B, Maeda J, et al. Imaging of Tau Pathology in a Tauopathy Mouse Model and in Alzheimer Patients Compared to Normal Controls. Neuron. 2013;79:1094-108.

19. Harada R, Okamura N, Furumoto S, Furukawa K, Ishiki A, Tomita N, et al. 18FTHK5351: A Novel PET Radiotracer for Imaging Neurofibrillary Pathology in Alzheimer Disease. J Nucl Med. 2016;57:208-14.

20. Ikari Y, Nishio T, Makishi Y, Miya Y, Ito K, Koeppe RA, et al. Head motion evaluation and correction for PET scans with 18F-FDG in the Japanese Alzheimer's disease neuroimaging initiative (J-ADNI) multi-center study. Ann Nucl Med. 2012;26:535-44. doi: 10.1007/s12149-012-0605-4

21. Welch BL. The Generalization of 'Student's' Problem when Several Different Population Variances are Involved. Biometrika. Oxford University PressBiometrika Trust; 1947;34:28. 22. McGill R, Tukey JW, Larsen WA. Variations of Box Plots. Am Stat. 1978;32:12.

23. Kimura Y, Ichise M, Ito H, Shimada H, Ikoma Y, Seki C, et al. PET Quantification of Tau Pathology in Human Brain with 11C-PBB3. J Nucl Med. 2015;56:1359-65.

24. Okamura N, Harada R, Ishiki A, Kikuchi A, Nakamura T, Kudo Y. The development and validation of tau PET tracers: current status and future directions. Clin Transl Imaging. 2018;6:305-16. 
Table 1 Characteristics of individuals by tracers

\begin{tabular}{|c|c|c|c|c|c|}
\hline Radiotracer & & $\begin{array}{c}{ }^{11} \mathrm{C}-\mathrm{PiB} \\
\text { (Amyloid) }\end{array}$ & $\begin{array}{c}{ }^{18} \text { F-florbetapir } \\
\text { (Amyloid) }\end{array}$ & $\begin{array}{c}{ }^{11} \text { C-PBB3 } \\
\text { (Tau) }\end{array}$ & $\begin{array}{c}{ }^{18} \text { F-THK5351 } \\
\text { (Tau) }\end{array}$ \\
\hline \# of subjects & & $\mathrm{n}=25$ & $\mathrm{n}=4$ & $\mathrm{n}=22$ & $\mathrm{n}=18$ \\
\hline \multirow[t]{2}{*}{ Age, years } & Median (IQR) & $73(65,79)$ & $71(67,73)$ & $70(60,75)$ & $76(69,81)$ \\
\hline & Min, Max & 49,87 & 59,73 & 47,81 & 55,85 \\
\hline Male gender & & $11(44 \%)$ & $1(25 \%)$ & $14(64 \%)$ & $7(39 \%)$ \\
\hline Positive & & $11(44 \%)$ & $2(50 \%)$ & $11(50 \%)$ & $13(72 \%)$ \\
\hline
\end{tabular}

Table 2 Perturbed transformation parameters

\begin{tabular}{|c|c|c|c|c|c|c|}
\hline & $\mathrm{x}[\mathrm{mm}]$ & $\mathrm{y}[\mathrm{mm}]$ & $\mathrm{z}[\mathrm{mm}]$ & $\operatorname{rot} \mathrm{x}[\mathrm{deg}]$ & rot y [deg] & $\operatorname{rot} \mathrm{z}$ [deg] \\
\hline Bas & 2.2 & 2.8 & 3.1 & 1.5 & 1.1 & 2.1 \\
\hline Maximum & 3.5 & 5.9 & 12.6 & 4.1 & 2.1 & 5.2 \\
\hline
\end{tabular}




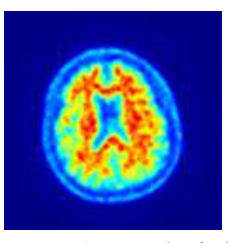

Tau or Amyloid PET

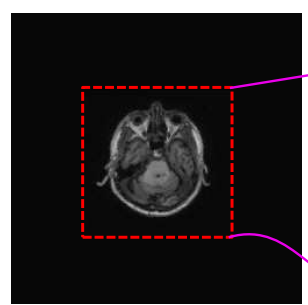

(a) Preprocessing

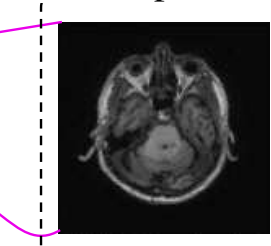

Resize \&

T1 weighted MRI
Trimming

(d) Difference is

evaluated as registration error

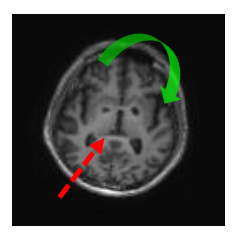

(b) Perturbed MRI

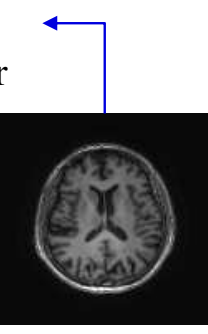

(c) Registration by SPM

Fig. 1 Summary of steps in the evaluation of registration accuracy

PiB Negative

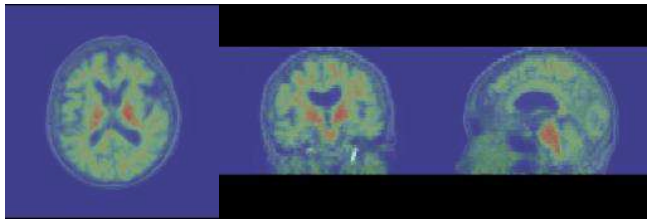

florbetapir Negative

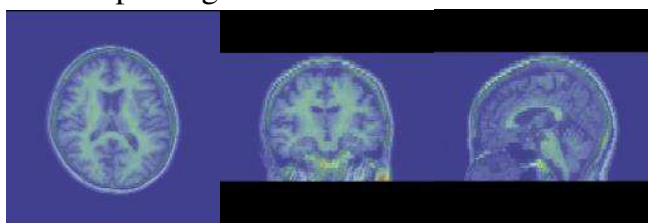

PBB3 Negative

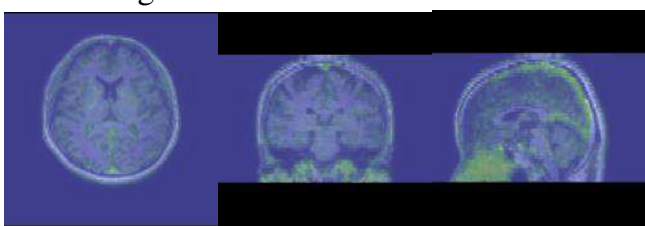

THK5351 Negative

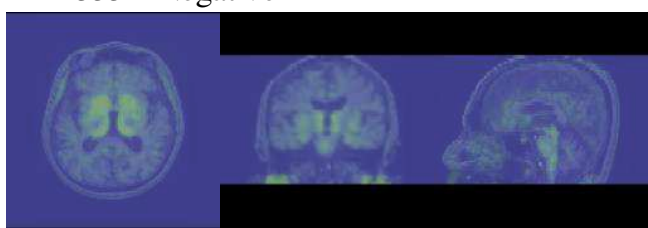

PiB Positive

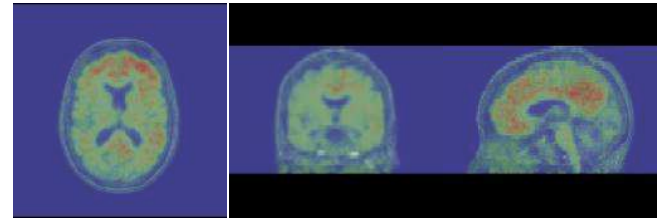

florbetapir Positive

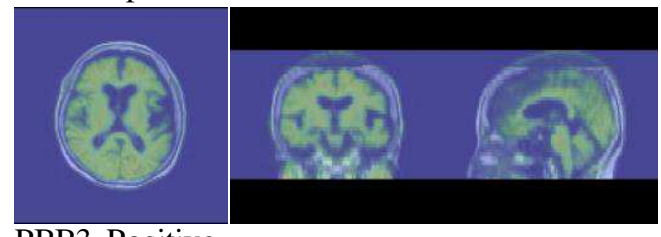

PBB3 Positive

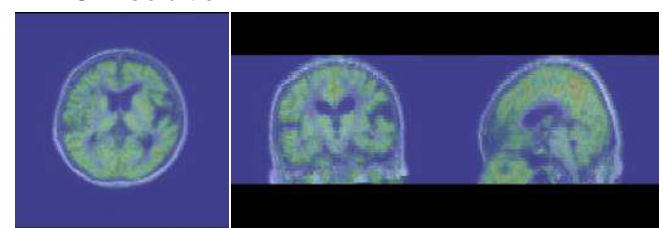

THK5351 Positive

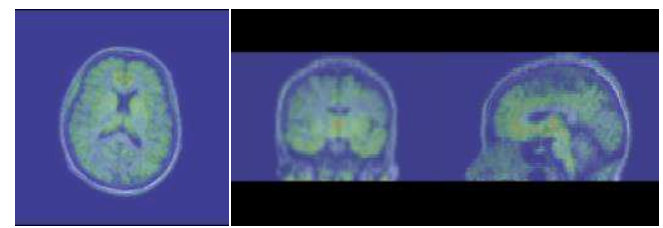

Fig. 2 Representative fusion images of PET and MRI after manual registration 
(a)

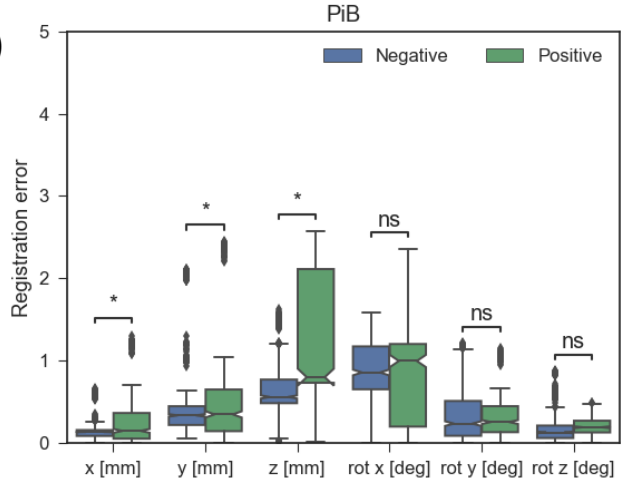

(c)

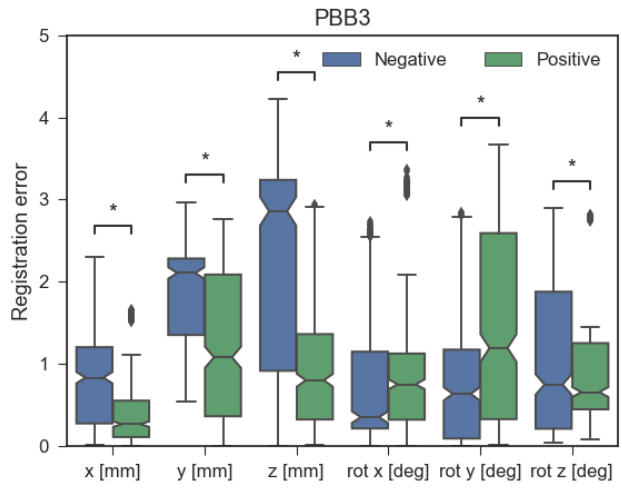

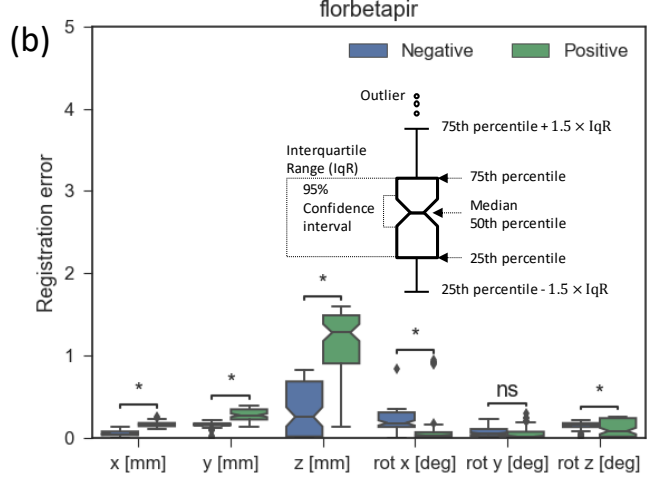

(d)

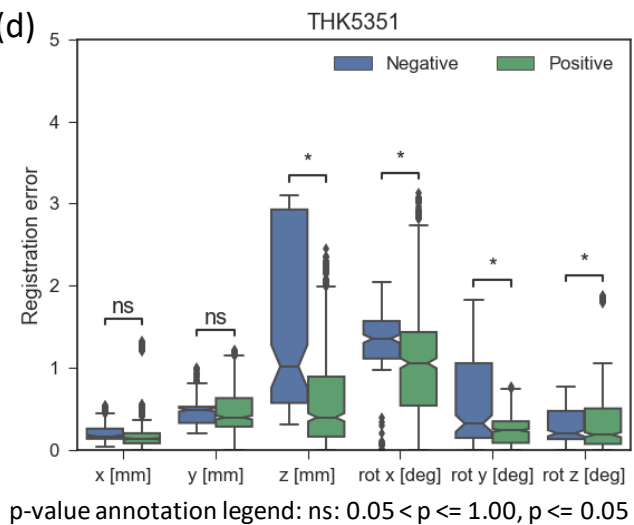

Fig. 3 Comparison of registration error, separated as positive or negative.

(a) PiB, (b) florbetapir, (c) PBB3 and (d) THK5351 

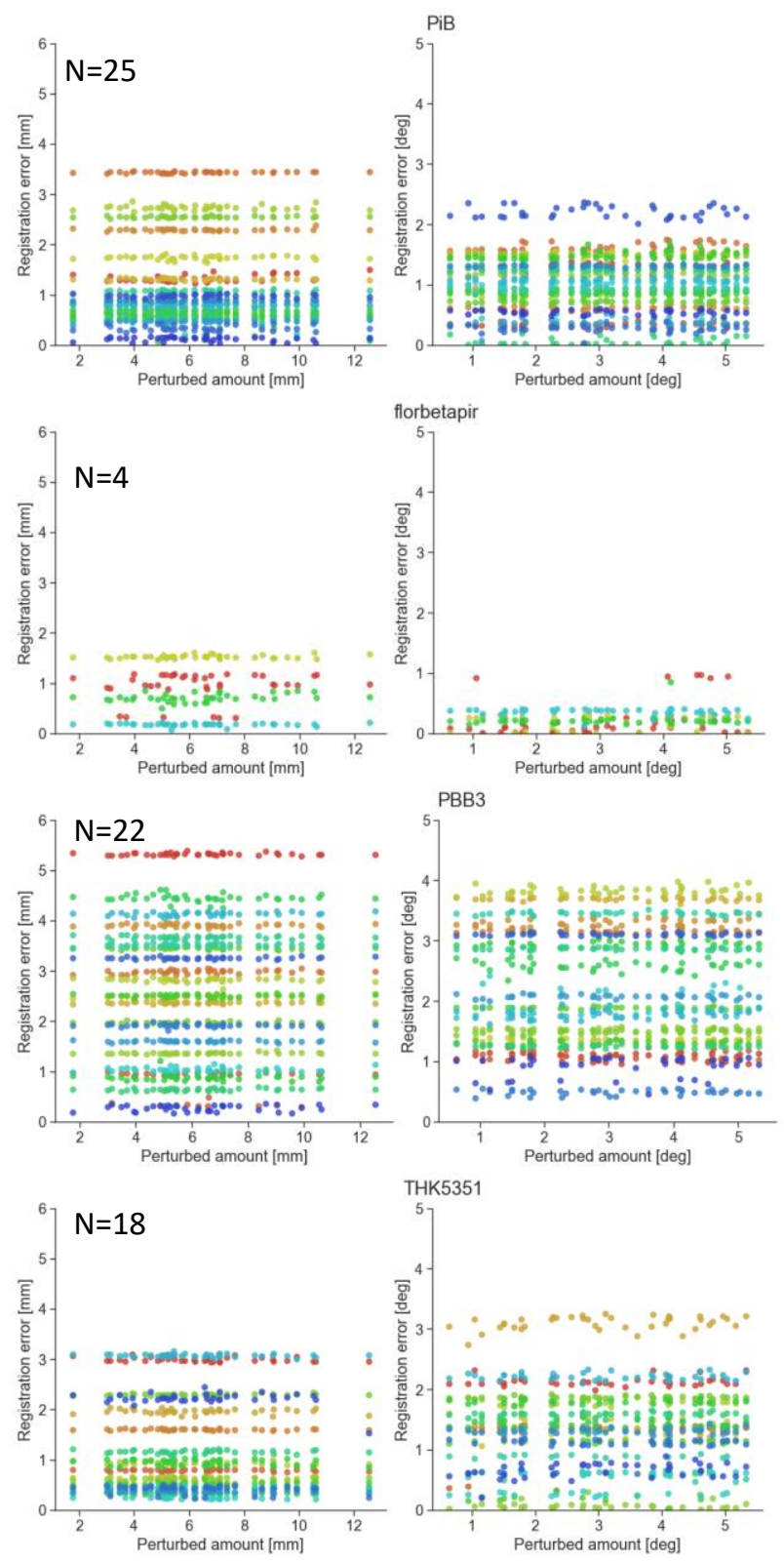

Fig. 4 Graphs plotting the perturbed parameter amount vs. registration error. The same color dots are data for the same subject: (a) PiB, (b) florbetapir, (c) PBB3 and (d) THK5351 
Figures

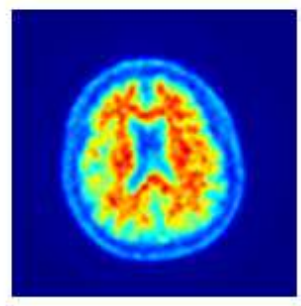

(d) Difference is

Tau or Amyloid PET

(a) Preprocessing ........... registration error

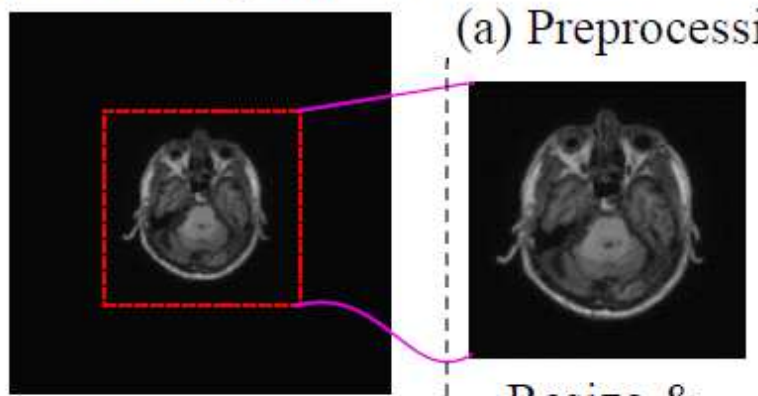

T1 weighted MRI
Resize \&

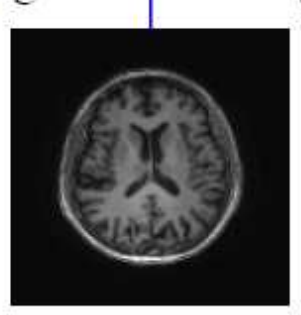

Manual Trimming alignment

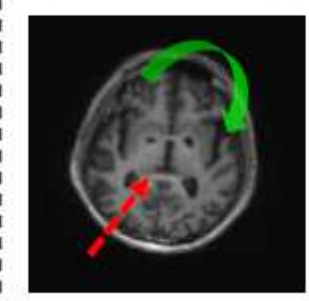

(b) Perturbed MRI

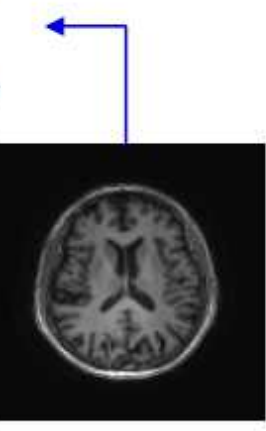

(c) Registration by SPM

Figure 1

Summary of steps in the evaluation of registration accuracy 
PiB Negative

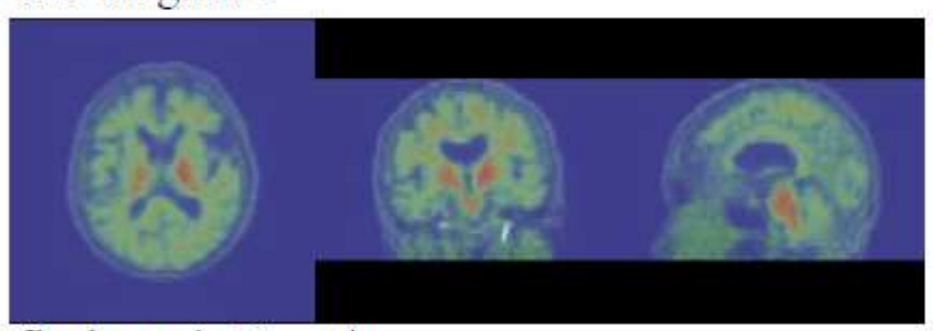

\section{florbetapir Negative}

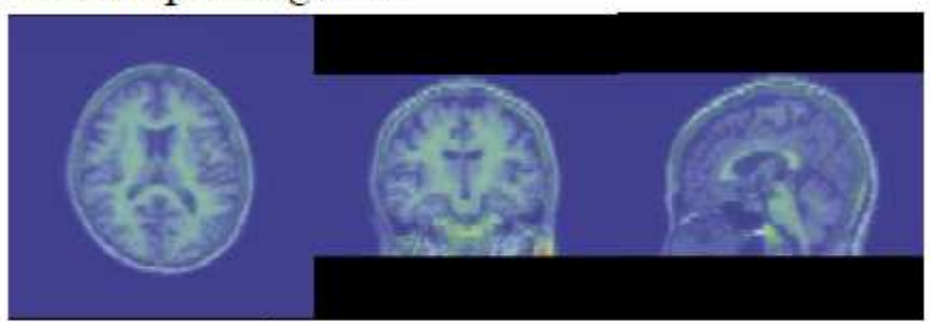

PBB3 Negative

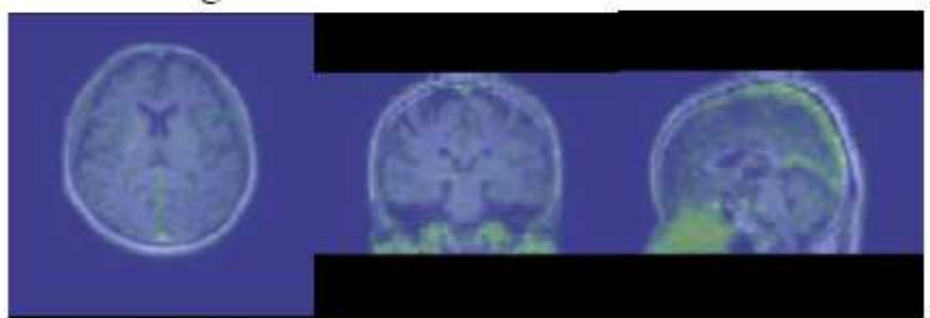

THK5351 Negative

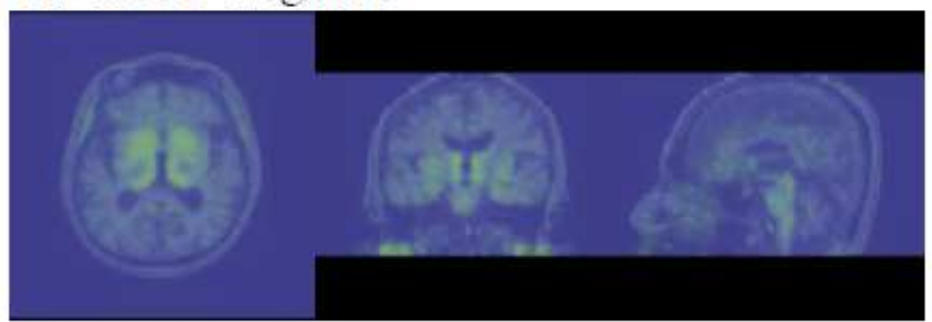

PiB Positive

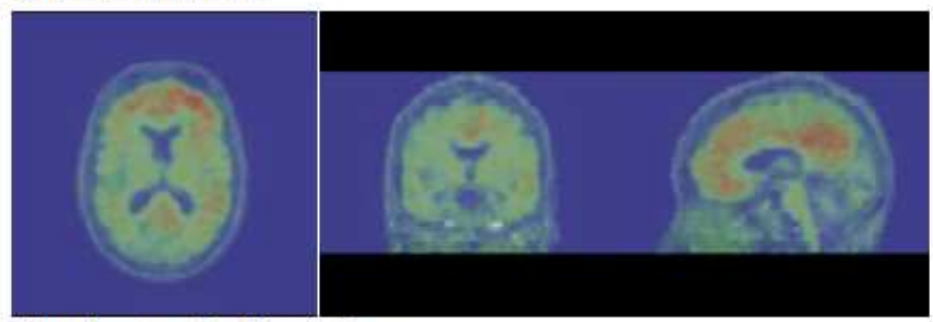

florbetapir Positive

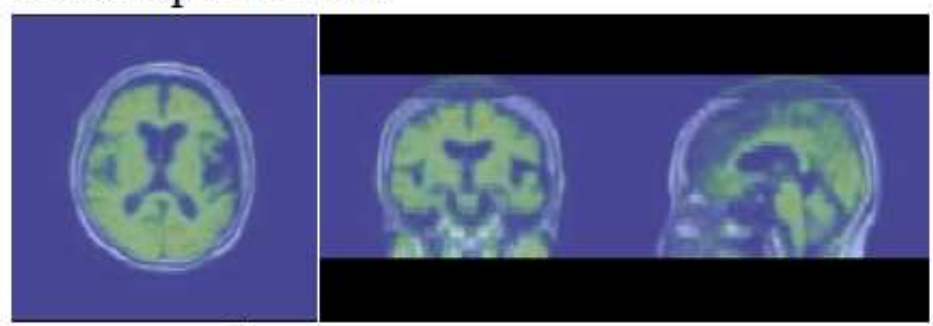

PBB3 Positive

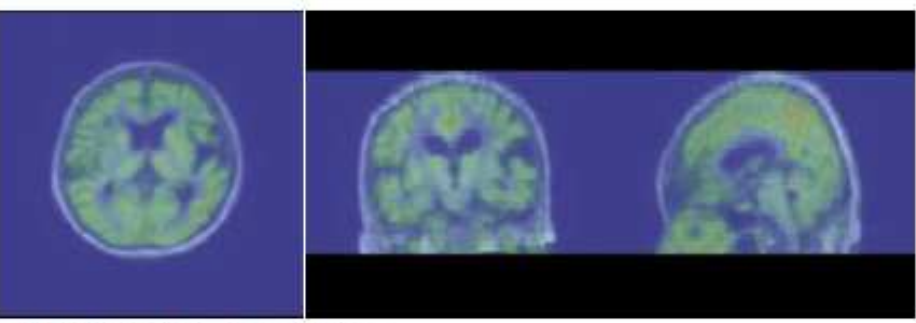

THK5351 Positive

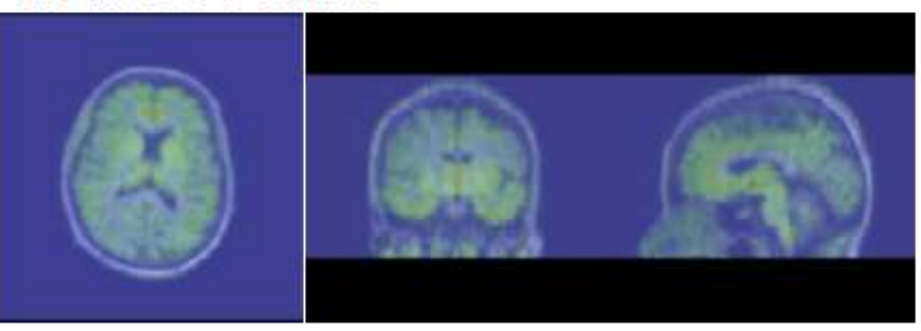

\section{Figure 2}

Representative fusion images of PET and MRI after manual registration 
(a)

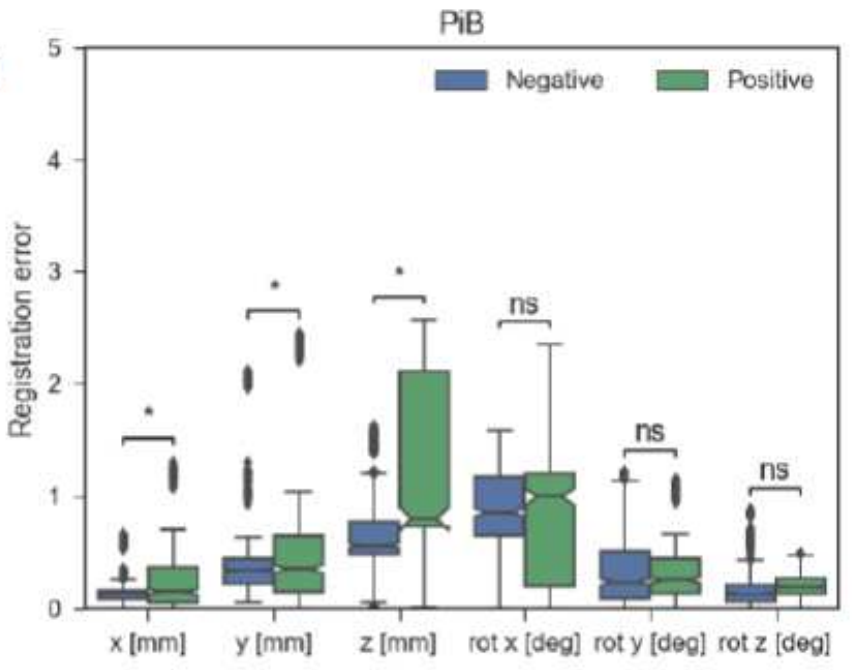

(c)

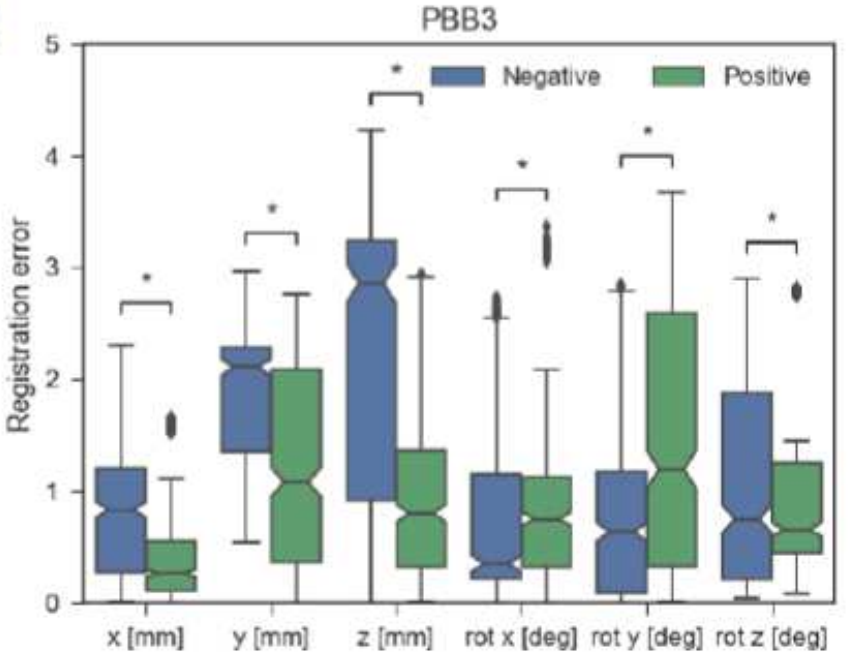

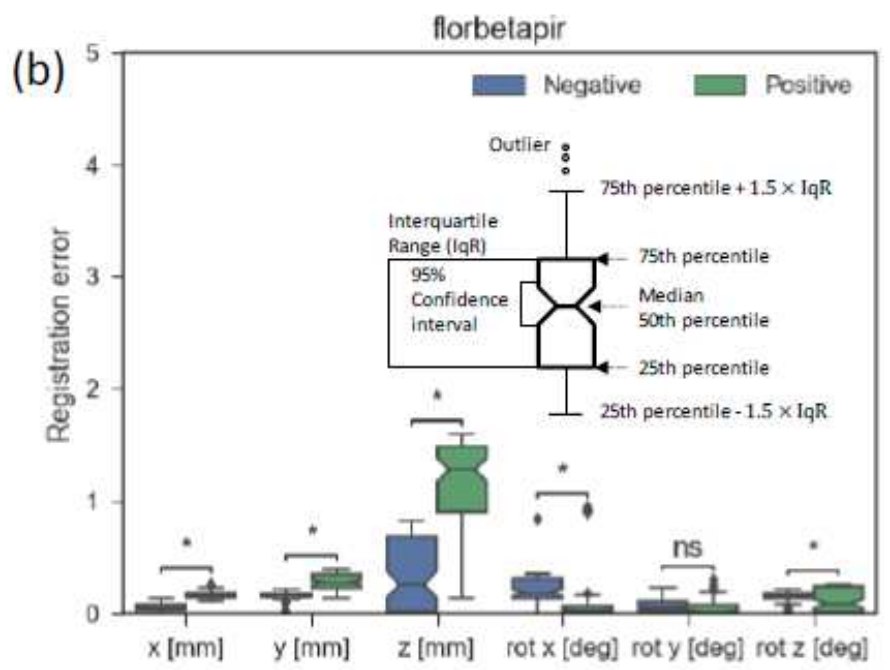

(d)

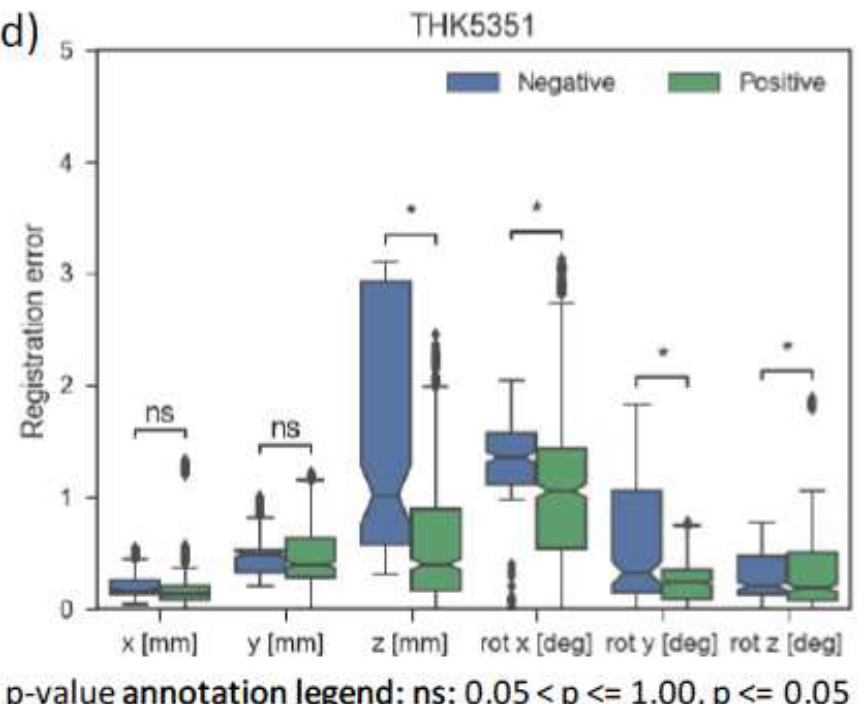

\section{Figure 3}

Comparison of registration error, separated as positive or negative. (a) PiB, (b) florbetapir, (c) PBB3 and (d) THK5351 

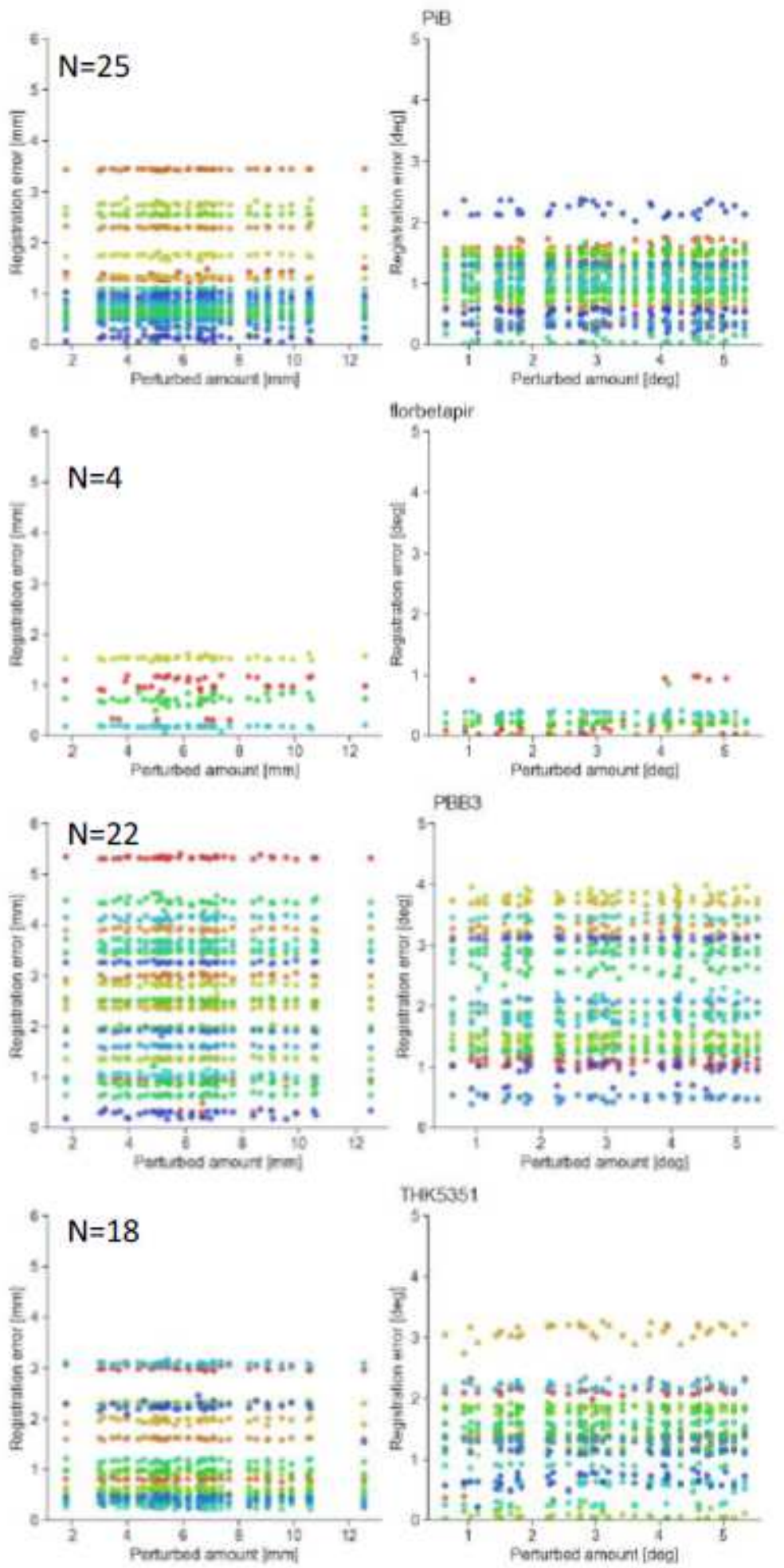

\section{Figure 4}

Graphs plotting the perturbed parameter amount vs. registration error. The same color dots are data for the same subject: (a) PiB, (b) florbetapir, (c) PBB3 and (d) THK5351

\section{Supplementary Files}


This is a list of supplementary files associated with this preprint. Click to download.

- EJNMMIElectronicsupplementarymaterialsubmit200131.pdf 